\title{
Single-institution retrospective review of elective and emergency embolization of renal angiomyolipoma
}

\author{
David Chapman $^{1}$; Matt Tyson ${ }^{1}$; Brendan Buckley ${ }^{2}$ \\ ${ }^{1}$ Department of Urology, Auckland City Hospital, Auckland, New Zealand; ${ }^{2}$ Department of Radiology, \\ Auckland City Hospital, Auckland, New Zealand
}

Cite as: Chapman D, Tyson M, Buckley B. Single-institution retrospective review of elective and emergency embolization of renal angiomyolipoma. Can Urol Assoc J 2021 May 11; Epub ahead of print. http://dx.doi.org/10.5489/cuaj.7143

Published online May 11, 2021

Correspondence: Dr. David Chapman, Department of Urology, Auckland City Hospital, Auckland, New Zealand; david_chapman@outlook.com

$* * *$

\section{Abstract}

Introduction: We aimed to evaluate the size reduction and complications after transcatheter embolization of renal angiomyolipomas (AMLs).

Methods: Cases from a single tertiary center were analyzed retrospectively. A blinded radiologist provided measurements of AMLs using a combination of ultrasound (US), computed tomography (CT), and magnetic resonance imaging (MRI). Electronic clinical notes, radiographic imaging, and laboratory data were reviewed.

Results: Twenty-one embolization procedures from 2002-2019 were analyzed. Four cases were emergency, the remainder elective. The average followup time after intervention was 42 months. Techniques included ethanol, PVA, Gelfoam, Embosphere ${ }^{\circledR}$, Histacryl ${ }^{\circledR}$, and coils. The median diameter size of AMLs was $8.6 \mathrm{~cm}$ pre-procedure and $6.0 \mathrm{~cm}$ post-procedure. The median volume of AMLs was $200 \mathrm{cc}$ pre-procedure and $67 \mathrm{cc}$ post-procedure, with a median reduction in volume of $55 \%$. One case $(4.8 \%)$ had a re-embolization and three cases $(14.3 \%)$ proceeded with surgical management of the AML. No cases re-presented with bleeding. Post-embolization syndrome is common. Renal arterial dissection and renal abscess are infrequent complications ( $9 \%$ and $4.5 \%$, respectively). There was no treatment-based mortality.

Conclusions: Embolization for renal AMLs is an established, safe, and effective method of treatment and our series further supports that. Determining when to intervene and how long to follow up patients is an issue that has not been well-described; more research needs to be done in this area. 


\section{Introduction}

Renal angiomyolipomas (AML) are the most common benign renal neoplasms. The majority are sporadic but are also found in association with tuberous sclerosis complex (TSC). AMLs are more common in females with a $4: 1$ female to male predominance. The incidence is estimated at up to $0.4 \%$ of the population.[1,2]

Their composition is a mixture of fat, vascular tissue and muscle.[2] The majority are mixed, however a small proportion, less than 5\%, are classified as minimal fat AMLs.[3]

Arteries within AMLs are frequently bizarre and tortuous and classically have disordered smooth muscle and absent internal elastic membranes on microscopy. For this reason, aneurysm formation and haemorrhage can occur. Large AMLs can recruit extra blood supply from surrounding vessels, including renal, adrenal, ureteral, gonadal, phrenic and lumbar arteries.[4]

AMLs can present in several ways, including flank/loin pain, a palpable mass, and after haemorrhage. One third of patients with haemorrhage present with shock[5], and in this setting is one of the manifestations Wunderlich syndrome.[5,6]

The natural history of renal AML has been described by Bhatt et al who observed 471 AMLs. Of these, $91 \%$ did not grow or grew slowly $(0.02 \mathrm{~cm} /$ year $)$ over a median follow-up of 43 months. No difference in growth rate was observed for tumours less than or greater than $4 \mathrm{cms}$. TSC cases were more frequent among the $9 \%$ of AMLs found to grow more rapidly $(>0.25 \mathrm{~cm} /$ year).[7] Longer term active surveillance cohort studies of renal AMLs are scarce.[2]

The point at which AMLs should receive prophylactic intervention is not categorically stated. The previously recognised cut-off of $4 \mathrm{~cm}$ in diameter is open to debate. The latest European Association of Urology Guidelines (2019) recommend treatment in cases where there is pain, bleeding, pregnancy, inadequate access for emergency care or suspected malignancy. It is recommended large tumours should be treated; however, a specific threshold does not exist. Active surveillance is appropriate for most AML, and factors associated with delayed intervention include tumour size $>4 \mathrm{~cm}$ and symptoms at diagnosis. The evidence behind this recommendation is classified as weak.[8,9] Similarly, Canadian Urology Association recent guidelines (2020) note most AMLs are asymptomatic and are at low risk of rupture. They recommend discussion of elective treatment in AMLs $>4 \mathrm{~cm}$.[10] Other factors must be taken into account as well, including aneurysmal component, pregnancy, coagulopathy, trauma, hormone level and co-morbidity with TSC.[11] The duration, imagining modality and which AMLs to follow up has no clear guidance. Treatment options include percutaneous intervention via selective arterial embolization (SAE), nephron sparing surgery and nephrectomy. Patients with TSC can be offered mTOR inhibitors. 
In this study, we retrospectively reviewed the acute and elective renal angiomyolipoma requiring embolization at a tertiary referral center over the last 20 years. We reviewed the size of AML for embolization and then the followup imaging of the AML and whether any patients required re-treatment or alternate treatment with surgery .

\section{Methods}

Cases from a single institution were analyzed retrospectively. Local ethical board approval was obtained. Cases were found using a systematic search of the hospital PACS system dating from January 2002 to June 2019 using the search terms "renal" or "kidney" and "embolization". From this list, cases relating to AML were reviewed. Only cases which involved SAE of an AML and had follow-up imaging were included in the statistical analysis.

Electronic clinical notes, radiographic imaging and laboratory data were reviewed. Demographic data (age, gender, and ethnicity), AML type (sporadic or TSC-associated), intervention type (elective or emergency), readmissions, complications, renal function, method of SAE, repeat SAE and progress to surgery was tabled. Pre-procedure imaging modalities for providing AML measurement include computed tomography (CT) and magnetic resonance imaging (MRI) with the latest imaging prior to intervention used. Postprocedure imaging modalities included ultrasound (US), CT and MRI .A blinded radiologist re-reviewed the imaging to provide the AML measurements over a one-week period. Volume was calculated using the ellipsoid formula $(\mathrm{H} \times \mathrm{W} \times \mathrm{AP} \times \pi / 6)$. Statistical analysis was performed using SAS 9.4 software by the institution's statistics department.

The decision to treat a patient was based on multidisciplinary input, involving urologists, interventional radiologists, and/or nephrologists/oncologists. Because the patients were gathered over 18 years, multiple clinicians were involved in the care of the patients. The procedure was performed under local anaesthesia via a common femoral artery approach. Various embolic agents were used which included polyvinyl alcohol (PVA), histoacryl glue, coils, gelfoam, Embozene, Embospheres and ethanol. Patients were admitted to the urology department for overnight admission and routine monitoring.

\section{Results}

A total of 25 individual patients were identified using the search technique, undergoing a total of 26 embolization procedures between them .Patients without follow-up or who underwent arteriography without embolization were excluded from the statistical analysis. The excluded procedures included three without follow-up imaging and two complicated by renal artery dissection which did not receive embolization. Figure 1.

One patient had three SAE procedures. The index procedure was a single AML SAE. The second procedure involved the aforementioned AML and a contralateral AML. The reembolization results were not included in the analysis.

The final statistical analysis included 20 patients undergoing 21 SAE procedures. 
Baseline demographics for all cases who received SAE and included in the analysis are outlined in table 1.

The primary embolization techniques include polyvinvyl alcohol $(\mathrm{n}=9,42.9 \%)$, Histoacryl $(n=5,23.8 \%)$, coils $(n=2,9.5 \%)$, ethanol $(n=2,9.5 \%)$, Embozene/sphere $(n=2$, $95 \%)$ and Gelfoam $(n=1,4.8 \%)$. Mean follow-up time was 42 months (range 1.9 - 172 months).

The image modality used for the final measurement for analysis was ultrasound in $23.8 \%(n=5)$, CT in $66.7 \%(n=13)$ and MRI in $14.2 \%(n=3)$. There was no fixed protocol. Emergency procedures accounted for four SAE procedures (19\%) and involved acute presentations with symptomatic and radiographically confirmed ruptured or bleeding AMLs. There were two additional cases with radiographic evidence of retroperitoneal haemorrhage which received SAE electively. In the elective cases, 4/17 (23.5\%) had pain prompting their index scan diagnosing an AML. The remainder were on active surveillance because of TSC status or incidentally identified sporadic AMLs. All SAEs were on AMLs $>4 \mathrm{~cm}$. In emergency cases, the median diameter was $12.3 \mathrm{~cm}$, range $4.7-22.2 \mathrm{~cm}$. In elective cases the median was $8.5 \mathrm{~cm}$, range $4.1-17.9 \mathrm{~cm}$. See table 2 .

Mean creatinine $(\mathrm{Cr})$ pre-embolization was $76 \mathrm{umol} / \mathrm{L}$ compared to $83 \mathrm{umol} / \mathrm{L}$ day 1 post-embolization with no statistical difference between the two groups. Maximum $\mathrm{Cr}$ was $148 \mathrm{umol} / \mathrm{L}$ pre and $178 \mathrm{umol} / \mathrm{L}$ post. The renal function of the patient who had bilateral and repeat embolization was unchanged pre and post.

All SAEs included in the analysis were deemed technically successful by the performing radiologist. In six cases (28.5\%), the performing radiologist reported insignificant residual flow within the tumour, the remaining reported complete cessation of flow within the tumour. See table 3.

There was a statistically significant decrease in volume post-embolization ( $\mathrm{p} 0.002)$ with a median of $200 \mathrm{ml}$ pre compared to $67 \mathrm{ml}$ post. There was a statistically significant decrease in diameter post-embolization $(\mathrm{p}<0.001)$ with a median of $8.6 \mathrm{~cm}$ pre compared to $6.0 \mathrm{~cm}$ post. The median percentage volume decrease post-embolization was $54.5 \%$. Median values have been reported as the results showed a skewed distribution. See table 4.

No patients were readmitted to the major hospitals in the region with bleeding AML or flank pain during the study period.

\section{Re-embolizations}

One patient had a re-embolization of the same AML, representing 4.8\% of AMLs and 4.5\% of patients. The indication for this was a persistent arterial feeder and a small increase in size was evident on imaging during workup for an enlarging contralateral AML. The time to reembolization was 15 years, 11 months.

\section{Surgical intervention}

Three patients proceeded to surgical management of same AML which had received SAE. This represents $14.3 \%$ of AMLs and $13.6 \%$ patients. 
Factors leading to surgical intervention included young age, future pregnancies, and minimal size change or increasing size. One had increase in size, going from $8.5 \mathrm{~cm}$ to $9.1 \mathrm{~cm}$ and given future planned pregnancies proceeded with surgery. The second had minimal size change, decreasing from $12.5 \mathrm{~cm}$ to $12.3 \mathrm{~cm}$ and ongoing flank pain, prompting surgical treatment. The third had an initial decrease of $1.9 \mathrm{~cm}$ from $12.4 \mathrm{~cm}$, with subsequent growth to $11.7 \mathrm{~cm}$. All were female under 45 years old.

The absolute post-procedure volume was the only statistically significant association to subsequent surgical management, with a median post volume size of $241 \mathrm{ml}$ in those going on to surgery vs $55 \mathrm{ml}$ who did not. The pre-procedure volume, relative volume change and change in diameter were not associated with proceeding to surgery .

\section{Complications}

Overall, the complication rate was low. There were three Grade III Clavien-Dindo complications. Two cases resulted in dissection of an intra-renal artery. These two cases were included in the complication rate but not in any other analysis of SAE outcomes, as the intention was to review results of those successfully treated by embolization. One was the embolization of a $5.9 \mathrm{~cm}$ AML in a patient with TSC which involved dissection of the superior segmental branch arising from right renal artery resulting in a functional occlusion. Secondary intraluminal thrombus was evident and cleared with a coaxial aspiration thrombectomy catheter .No other treatment was required, and follow-up imaging showed stable size. The second was the embolization of $3.6 \mathrm{~cm}$ AML which bled after thrombolysis and resulted in dissection of a medial vessel branch that supplied the AML, stopping blood flow to the AML. No other treatment during the procedure was required .One patient developed a peri-renal abscess requiring percutaneous drainage resulting in prolonged admission.

Five grade I Clavien-Dindo complications occurred. These were all post-embolization syndrome, defined as fever, abdominal pain, nausea or vomiting. These were all managed conservatively with analgesia, anti-pyretics and anti-emetics.

No treatment-based mortality was identified.

\section{Discussion}

Embolization for renal AMLs is an established safe and effective method of treatment.[1214]. Our study shows a significant decrease in volume and maximal diameter postembolization, with a median decrease in size of $55 \%$. There were no episodes of rebleeding during the study follow-up time, and only a small proportion required surgical management or re-embolization.

Overall complication rate was low, consistent with other recent series.[13,15,16] Minor complications included post embolization syndrome in $24 \%$ of patients. Three major complications occurred. Two cases resulted in catheter or wire related dissection of an intrarenal artery which provided a functional occlusion to the tumour in both cases and were managed conservatively. One patient developed a peri-renal abscess requiring percutaneous 
drainage, an infrequent complication noted in other series.[13,17] No treatment-based mortality was detected during the study period.

The lack of prospective, randomized studies in the management of AMLs and the significant heterogeneity in the available retrospective evidence presents a challenge in many aspects of their clinical management.[10] Current evidence shows that the chance of becoming symptomatic from an AML increases significantly with size greater than $4 \mathrm{~cm}$ or growth rate greater than $0.25 \mathrm{~cm} / y e a r$. The traditional cut-off has been $4 \mathrm{~cm}$ before intervention, however some now recommend not intervening until size is greater than $6 \mathrm{~cm}$.[18] Using size as the only parameter for intervention is an overly simplified approach and other factors which should be included in decision making include size of aneurysmal component and multi-focal tumours, TSC status, pregnancy status, patient preference, coagulopathy, trauma, access to emergency services, availability of surveillance imaging and renal function.[11,18]

Using maximal diameter as a predictor of rupture, one study showed that using a cutoff of $4 \mathrm{~cm}$ resulted in a sensitivity of $100 \%$ and specificity of $38 \%$. If a cut-off of $6 \mathrm{~cm}$ is used, the sensitivity is $100 \%$ and specificity is $67 \%$. Using an aneurysmal component of greater than $5 \mathrm{~mm}$ as a predictor of rupture is more accurate again with sensitivity of $100 \%$ and specificity of $86 \%$. [19] We did not measure the largest aneurysmal component in out series. Kuusk et al has shown $74 \%$ of haemorrhagic AMLs are greater than $6 \mathrm{~cm}$.[20] Less than $6 \mathrm{~cm}$ AMLs are still at risk of rupture, however to prophylactically treat all AMLs greater than $4 \mathrm{~cm}$ would overtreat up to $65 \%$ of patients. [18] Ouzid et al showed that of 130 patients on active surveillance, $13 \%(\mathrm{n}=17)$ proceeded to intervention. In tumours $>4 \mathrm{~cm}, 34 \%(\mathrm{n}=13)$ failed active surveillance, with only a small proportion due to haemorrhage. [18] Other studies have shown $9.4 \%$ of $<4 \mathrm{~cm}$ AMLs rupture [21] and AMLS $>10 \mathrm{~cm}$ can safely receive active surveillance [22] indicating both ends of the spectrum can behave unexpectedly from previous thinking. Neither the most recent Canadian nor European Urology Association guidelines recommend a specific size threshold for treatment. [8,10]

In our series, emergency cases receiving SAE had a mean diameter of $12.3 \mathrm{~cm}$ and the smallest tumour by diameter was $4.7 \mathrm{~cm}$, with the remainder greater than $9 \mathrm{~cm}$.This was similar for patients with pre-intervention evidence of retroperitoneal haemorrhage. Our study is not designed or powered to make statements on elective thresholds for treatment. However, our data suggests if $6 \mathrm{~cm}$ was the threshold, $75 \%$ of emergency AMLs would have been electively treated.

There was variation in follow-up imaging. MR has been proposed as the superior imaging modality, especially in the case of fat poor AML and cumulative radiation exposure.[23] However, time and resource constraints around the use of MR often mean this is not practical. There are no firm guidelines regarding appropriate follow-up of treated AMLs.

Limitations of this study include the retrospective design with no randomisation or control groups. A major limitation is the variation of embolization materials which limits generalisability and given the numbers, no statistical analysis between these groups could be 
undertaken. There was a limited number of interventions and no standardised follow-up. A significant proportion (24\%) of post treatment imaging was based on US rather than crosssectional modalities. This may have affected the reliability of the results as a comparison was being made across imaging modalities. Of note, none of the patients proceeding with surgical management had US as their most recent imaging modality prior to surgery. Despite blinding of the radiologist, evidence of treatment (eg coils), would have been apparent and may have introduced bias. Furthermore, the radiologist is limited in the interpretation of US images to those that have been saved.

Further research is needed to clarify when intervention is required, particularly for AMLs within the $4-6 \mathrm{~cm}$ size range. The length of follow-up and imaging modality needs further study.

\section{Conclusions}

Embolization for renal AMLs is an established safe and effective method of treatment and our series further supports this. Our series showed no rebleeds, low complication rates and only a small proportion proceeding with surgical management. Determining when to intervene and how long to follow up patients is an issue that has not been well described, with more research needing to be done in this area. 


\section{References}

1. Fujii Y, Ajima J-I, Oka K, et al. Benign renal tumors detected among healthy adults by abdominal ultrasonography. European urology. 1995;27:124-127.

2. Nelson CP, Sanda MG. Contemporary diagnosis and management of renal angiomyolipoma. The Journal of urology. 2002;168(4):1315-1325.

3. Kim JK, Park S-Y, Shon J-H, et al. Angiomyolipoma with minimal fat: differentiation from renal cell carcinoma at biphasic helical CT. Radiology. 2004;230(3):677-684.

4. Yamakado K, Tanaka N, Nakagawa T, et al. Renal angiomyolipoma: relationships between tumor size, aneurysm formation, and rupture. Radiology. 2002;225(1):78-82.

5. Dickinson M, Ruckle H, Beaghler M, et al. Renal angiomyolipoma: optimal treatment based on size and symptoms. Clinical nephrology. 1998;49(5):281-286.

6. Katabathina VS, Katre R, Prasad SR, et al. Wunderlich syndrome: cross-sectional imaging review. Journal of computer assisted tomography. 2011;35(4):425-433.

7. Bhatt JR, Richard PO, Kim NS, et al. Natural history of renal angiomyolipoma (AML): most patients with large AMLs $>4 \mathrm{~cm}$ can be offered active surveillance as an initial management strategy. European urology. 2016;70(1):85-90.

8. Ljungberg B, Albiges L, Bensalah K, et al. EAU Guidelines on Renal Cell Carcinoma. ISBN 978-94-92671-07-3. http://uroweb.org/guidelines/compilations-ofall-guidelines/. European association of urology. 2020 [cited 73 p.].

9. Fernández-Pello S, Hora M, Kuusk T, et al. Management of sporadic renal angiomyolipomas: a systematic review of available evidence to guide recommendations from the European Association of Urology Renal Cell Carcinoma Guidelines Panel. European urology oncology. 2020;3(1):57-72.

10. Guo Y, Kapoor A, Cheon P, et al. Canadian Urological Association best practice report: Diagnosis and management of sporadic angiomyolipomas. Canadian Urological Association Journal. 2020;14(11):E527.

11. Wang C, Li X, Peng L, et al. An update on recent developments in rupture of renal angiomyolipoma. Medicine. 2018;97(16).

12. Ramon J, Rimon U, Garniek A, et al. Renal angiomyolipoma: long-term results following selective arterial embolization. European urology. 2009;55(5):1155-1162.

13. Bardin F, Chevallier O, Bertaut A, et al. Selective arterial embolization of symptomatic and asymptomatic renal angiomyolipomas: a retrospective study of safety, outcomes and tumor size reduction. Quantitative imaging in medicine and surgery. 2017;7(1):8.

14. Kothary N, Soulen MC, Clark TW, et al. Renal angiomyolipoma: long-term results after arterial embolization. Journal of Vascular and Interventional Radiology. 2005;16(1):45-50.

15. Duan X-H, Zhang M-F, Ren J-Z, et al. Urgent transcatheter arterial embolization for the treatment of ruptured renal angiomyolipoma with spontaneous hemorrhage. Acta Radiologica. 2016;57(11):1360-1365.

16. Hocquelet A, Cornelis F, Le Bras Y, et al. Long-term results of preventive embolization of renal angiomyolipomas: evaluation of predictive factors of volume decrease. European radiology. 2014;24(8):1785-1793.

17. Özkara H, Özkan B, Solok V. Management of renal abscess formation after embolization due to renal angiomyolipomas in two cases. International urology and nephrology. 2006;38(3-4):427-429. 
18. Ouzaid I, Autorino R, Fatica R, et al. Active surveillance for renal angiomyolipoma: outcomes and factors predictive of delayed intervention. BJU international. 2014;114(3):412-417.

19. Ryan JW, Farrelly C, Geoghegan T. What Are the Indications for Prophylactic Embolization of Renal Angiomyolipomas? A Review of the Current Evidence in the Literature. Canadian Association of Radiologists Journal. 2018;69(3):236-239.

20. Kuusk T, Biancari F, Lane B, et al. Treatment of renal angiomyolipoma: pooled analysis of individual patient data. BMC urology. 2015;15(1):123.

21. Prischl FC, Spöttl P. Spontaneous rupture of angiomyolipoma of the kidney. Wiener klinische Wochenschrift. 2017;129(5-6):217-218.

22. Gomha M, Al-Gahwary M, Alsowayan Y. PD73-09 big renal angiomyolipoma: the 4 $\mathrm{cm}$ size limit for conservative management should be revisited? The Journal of Urology. 2017;197(4S):e1370-e1370.

23. Krueger DA, Northrup H, Roberds S, et al. Tuberous sclerosis complex surveillance and management: recommendations of the 2012 International Tuberous Sclerosis Complex Consensus Conference. Pediatric neurology. 2013;49(4):255-265. 


\section{Figures and Tables}

\begin{tabular}{|l|c|}
\hline Table 1. Patient baseline demographics \\
\hline Variable & \\
\hline Gender, $\mathrm{n}(\%)$ & $15(75 \%)$ \\
\hline Female & $5(25 \%)$ \\
\hline Male & 54 \\
\hline Age (years) & $23-87$ \\
\hline Mean & \\
\hline Range & $2(10 \%)$ \\
\hline TSC, $\mathrm{( \% )}$ & $18(90 \%)$ \\
\hline Yes & \\
\hline No & $4(80 \%)$ \\
\hline Bilateral AML on imaging, $\mathrm{n}(\%)$ & $16(20 \%)$ \\
\hline Yes & \\
\hline No & $12(57 \%)$ \\
\hline Side of treated AML $\mathrm{n}(\%)$ & $9(43 \%)$ \\
\hline Left & \\
\hline Right & \\
\hline
\end{tabular}

AML: angiomyolipoma.

\begin{tabular}{|l|c|}
\hline \multicolumn{2}{|l|}{ Table 2. Tumour size before SAE } \\
\hline Variable & $\mathbf{n = 2 1}$ \\
\hline Size of all AMLs before SAE, cm & $9.1 \pm 4.4$ \\
\hline Mean \pm SD & $\mathbf{n = 1 7}$ \\
\hline Median (range) & $8.6(4.1-22.2)$ \\
\hline $\begin{array}{l}\text { Size of electively treated AMLs before } \\
\text { SAE, cm }\end{array}$ & $8.5(4.1-17.9)$ \\
\hline Mean \pm SD & $\mathbf{n = 4}$ \\
\hline Median (range) & $12.3 \pm 6.4$ \\
\hline $\begin{array}{l}\text { Size of emergency treated AMLs before } \\
\text { SAE, cm }\end{array}$ & $11.1(4.7-22.2)$ \\
\hline Mean \pm SD & $\mathbf{n = 6}$ \\
\hline Median (range) & $12.7 \pm 5.8$ \\
\hline Size of ruptured AMLs before SAE, cm & $11.1(4.7-22.2)$ \\
\hline Mean \pm SD & Median (range)
\end{tabular}

AML: angiomyolipoma; SD: standard deviation; SAE: selective arterial embolization. 


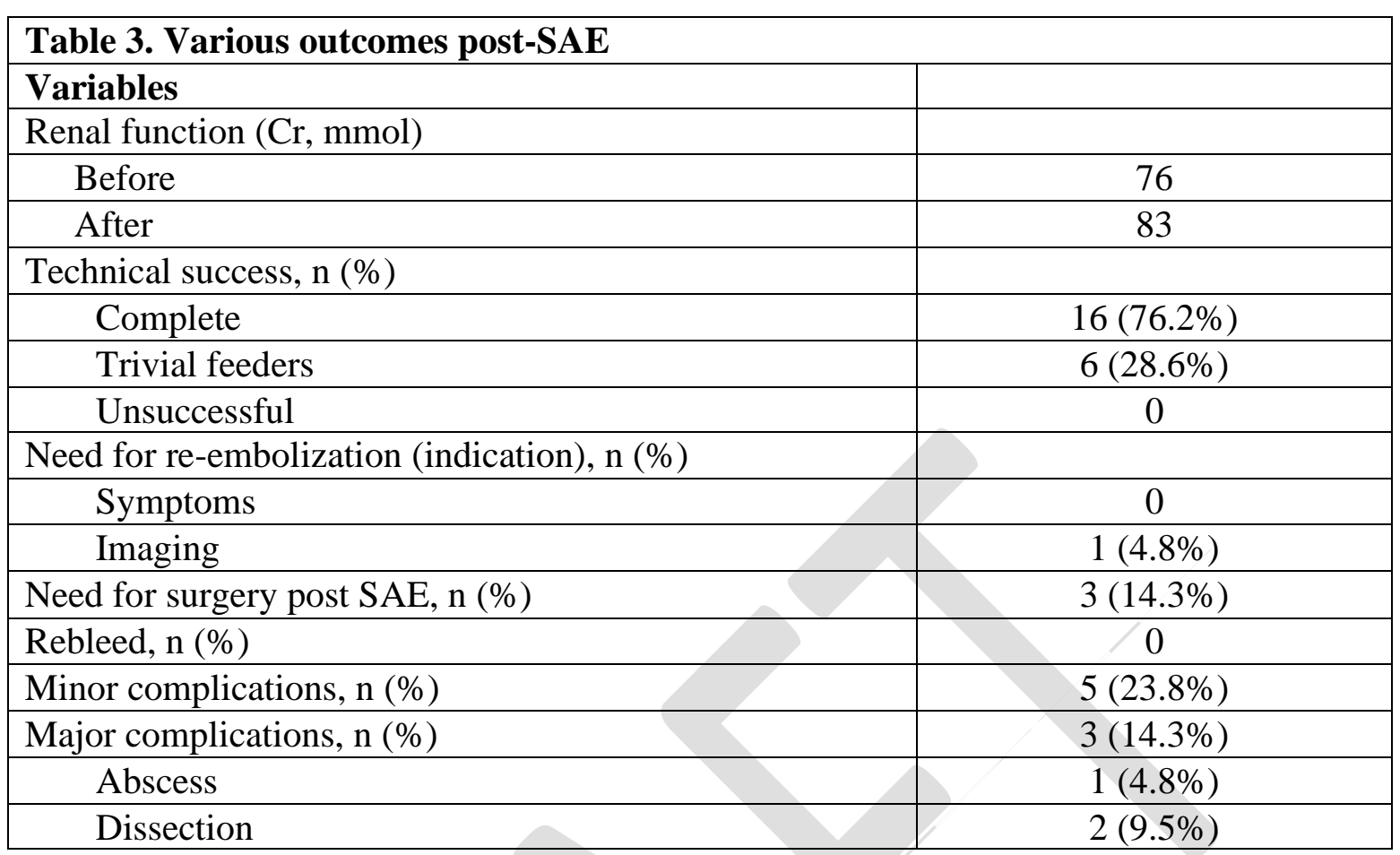

Cr: creatinine; SAE: selective arterial embolization.

\begin{tabular}{|c|c|c|c|c|c|c|}
\hline Variable & Minimum & Median & Maximum & $\begin{array}{c}\text { Lower } \\
95 \% \\
\text { mean }\end{array}$ & $\begin{array}{l}\text { Upper } \\
95 \% \\
\text { mean }\end{array}$ & $\mathbf{p}$ \\
\hline $\begin{array}{l}\text { Volume pre- } \\
\text { embolization }(\mathrm{ml})\end{array}$ & 23.6 & 200.3 & 2276.6 & 127.5 & 549.9 & \\
\hline $\begin{array}{l}\text { Volume post- } \\
\text { embolization }(\mathrm{ml})\end{array}$ & 3.48 & 67.1 & 753.0 & 79.9 & 273.4 & \\
\hline $\begin{array}{l}\text { Maximum diameter } \\
\text { pre-embolization } \\
(\mathrm{cm})\end{array}$ & 4.1 & 8.6 & 22.2 & 7.2 & 11.0 & \\
\hline $\begin{array}{l}\text { Maximum diameter } \\
\text { post-embolization } \\
(\mathrm{cm})\end{array}$ & 2.4 & 6.0 & 13.9 & 5.5 & 8.5 & \\
\hline $\begin{array}{l}\text { Diameter change } \\
(\mathrm{cm})\end{array}$ & -2.3 & 1.8 & 8.3 & 0.91 & 3.3 & 0.002 \\
\hline Volume decrease (\%) & -177.8 & 54.5 & 98.6 & 3.5 & 62.2 & 0.07 \\
\hline
\end{tabular}

SAE: selective arterial embolization. 